\title{
The cosmic web of dwarf galaxies in a warm versus cold dark matter universe: Mock galaxies in CDM and WDM simulations
}

\author{
Darren S. Reed*, Aurel Schneider, Doug Potter, Joachim Stadel, Ben Moore \\ Institute for Computational Science, Univ. of Zürich, \\ Winterthurerstrasse 190, CH-8057 Zürich, Switzerland \\ *E-mail: reed@physik.uzh.ch
}

Robert E. Smith

Astronomy Centre, Department of Physics and Astronomy, University of Sussex Group BN1 9QH, Brighton, United Kingdom

\begin{abstract}
Using cosmological simulations, we show that the cosmic web of dwarf galaxies in a warm dark matter (WDM) universe, wherein low mass halo formation is heavily suppressed, is nearly indistinguishable to that of a cold dark matter (CDM) universe whose low mass halos are not seen because galaxy formation is suppressed below some threshold mass. Low mass warm dark matter halos are suppressed nearly equally in all environments. For example, WDM voids in the galaxy distribution are neither larger nor emptier than CDM voids, once normalized to the same total galaxy number density and assuming galaxy luminosity scales with halo mass. It is thus a challenge to find hints about the dark matter particle in the cosmic web of galaxies. However, if the scatter between dwarf galaxy luminosity and halo properties is large, low mass CDM halos would sometimes host bright galaxies thereby populating voids that would be empty in WDM. Future surveys that will capture the small scale clustering in the local volume could thus help determine whether the CDM problem of the over-abundance of small halos with respect to the number density of observed dwarf galaxies has a cosmological solution or an astrophysical solution.
\end{abstract}

Keywords: Cosmology; dwarf galaxies; warm dark matter; MG14 Proceedings.

\section{Halos and Galaxies in Warm and Cold Dark Matter Cosmologies}

In WDM cosmological models, small scale power is suppressed, reducing the number of low mass dwarf galaxy halos. This brings dwarf galaxy numbers into agreement with the reduced number of satellite galaxies in the Milky Way and M31 relative to the large number of CDM satellite halos in simulations ${ }^{1,2}$, solving the missing satellite problem. Inefficient star and galaxy formation within low mass halos has also been proposed as a solution within CDM cosmology (e.g. Ref. 3). Hence either a cosmological solution (warm dark matter) or an astrophysical solution (baryon physics) has the potential to explain this CDM problem.

By design, the two classes of solutions are degenerate with each other in many ways since they both reduce visible small scale structures. We thus consider whether other clustering statistics might show some discriminating power. Voids, in particular, have often been noted to be larger and emptier in WDM than $\mathrm{CDM}^{4,5}$. However, since voids are delineated by galaxies (lying in halos), the sizes and emptiness of voids is highly sensitive to the number abundance of galaxies in a survey, as well 
as to the relation and scatter between halo mass and galaxy properties. We consider how these issues affect void statistics as a constraint for CDM versus WDM.

We use gravity-only numerical simulations to model two twin $25 \mathrm{Mpc}$ volumes, one with a $\Lambda$ CDM cosmology and one with a $\Lambda$ WDM cosmology, presented in Ref. 6 . Each volume is a periodic cube of $1024^{3}$ equal mass particles, for a particle mass of $3.9 \times 10^{5} h^{-1} M_{\odot}$. The $\Lambda \mathrm{WDM}$ assumes a $2 \mathrm{keV}$ thermal relic warm particle. Simulations are evolved using the particle gravity tree-code PKDGRAV ${ }^{7}$.

\subsection{Mock galaxy catalog}

We construct a mock galaxy catalog using halos identified by the Amiga Halo Finder $\left(\mathrm{AHF}^{8,9}\right)$, which finds self-bound halos and satellite halos (subhalos). Mock galaxies are matched by circular velocity $\left(V_{c}\right)$ at the peak of the rotation curve. The catalogs for each cosmology are matched to each other by galaxy abundance, necessary for a fair comparison of their clustering properties. We sample into the range of small-scale WDM suppression. At the minimum WDM $V_{c}$ of $13.7 \mathrm{~km} \mathrm{~s}^{-1}$, galaxy numbers are reduced by approximately a factor of ten. The CDM catalog extends only to $\mathrm{V}_{\mathrm{c}}>22.9 \mathrm{~km} \mathrm{~s}^{-1}$ to match the WDM galaxy abundance. Each catalog has approximately $10^{4}$ mock galaxies. Contamination from artificial WDM structure is small for the halo masses we include, and we apply an additional criteria based on the shape of the initial Lagrangian region of each halo to remove spurious WDM halos (See details in Ref. 6). We consider also the effects of large scatter between luminosity, $L$, and $V_{c}$.

\section{The Distribution of Dwarf Galaxies}

\subsection{With a one-to-one relation of luminosity versus halo $V_{c}$}

Fig. 1 slows a slice of the galaxy distribution from each mock catalog. In the case of no scatter between luminosity and $V_{c}$, the properties of the distribution of galaxies is visually indistinguishable in CDM and WDM. With no scatter in $L / V_{c}$, the self abundance-matched CDM and WDM catalogs are nearly identical for all statistics that we examined: the auto-correlation function; the numbers and radial profiles of satellites; the numbers of isolated galaxies; and the PDF of small voids. Thus, the cosmic web of CDM and WDM galaxies is essentially identical if one assumes that low mass CDM halos cannot host galaxies. WDM voids are not larger or "emptier" of halos than CDM, as is sometimes loosely stated, but are nearly identical if one links galaxy properties to halo properties by simple abundance matching.

\subsection{With scatter in luminosity versus halo $V_{c}$}

The situation changes, however, in the case of high $L / V_{c}$ scatter for dwarf galaxies. Differences become apparent in the cosmic web. We demonstrate this by allowing luminosity to scatter by up to 2.5 magnitudes (one dex in mass to light ratio) for 


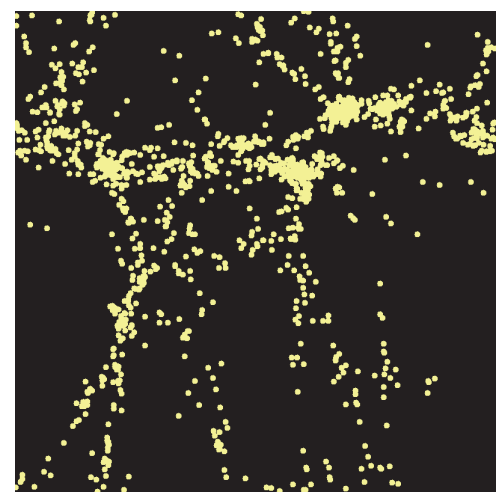

(a) CDM, No Scatter

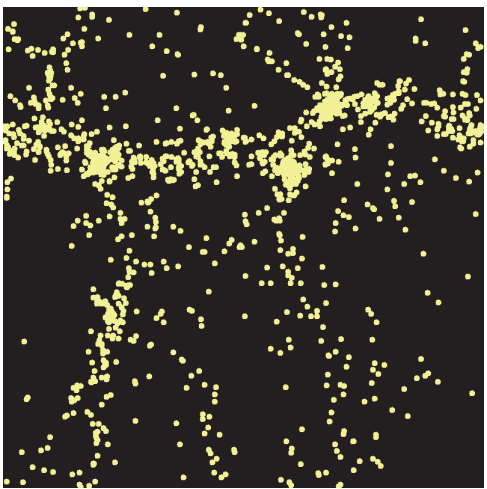

(c) CDM with Large $L / V_{c}$ Scatter

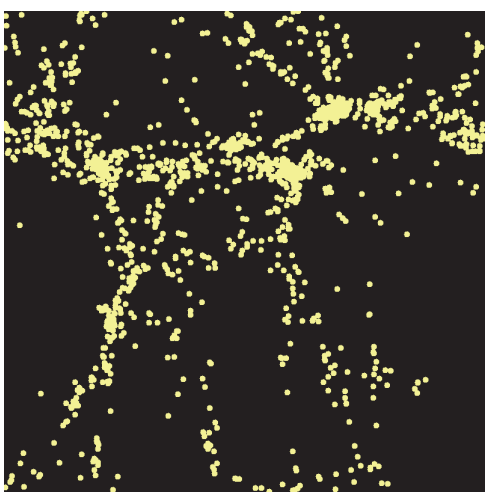

(b) WDM, No Scatter

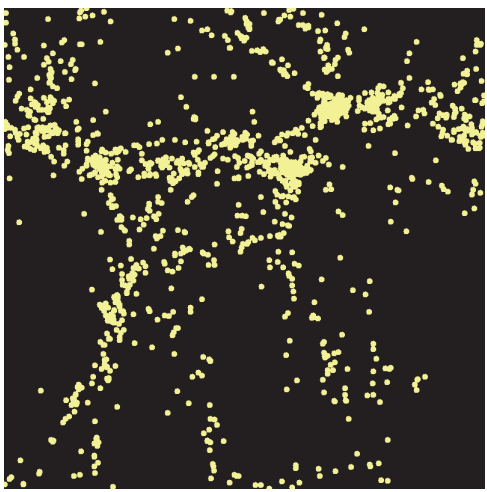

(d) WDM with Large $L / V_{c}$ Scatter

Fig. 1. $25 \mathrm{Mpc}$ wide slice of our simulation volume. Each point is a mock dwarf galaxy. Catalogs are constructed with different minimum $V_{c}$ selection thresholds so that the WDM and CDM catalogs contain the same number of galaxies. CDM and WDM are visually indistinguishable unless large scatter (one dex here) is assumed between dwarf galaxy luminosity and halo $V_{c}$. With large $L / V_{c}$ scatter, CDM voids become somewhat populated by galaxies hosted by low mass halos that have been scattered up to relatively high luminosity (panel c).

low mass halos by applying the following relation for the rms scatter in galaxy magnitude: $\sigma_{M a g}\left(V_{c}\right)=0.4+\left(V_{c} / 80 \mathrm{~km} \mathrm{~s}^{-1}\right)^{-2}$, and $\sigma_{M a g}\left(V_{c}\right)=2.5$ for $V_{c}<$ $55 \mathrm{~km} \mathrm{~s}^{-1}$; we set $\sigma_{M a g}\left(V_{c}\right)=0.4$ for $V_{c}>80 \mathrm{~km} \mathrm{~s}^{-1}$ This large scatter causes some low mass CDM halos in low density regions that were below the $V_{c}$ threshold for inclusion into the catalog to scatter up in luminosity such that voids become sparsely populated with relatively isolated dwarfs; this is apparent in panel c of 
Fig. 1. The $L / V_{c}$ scatter has no noticeable effect in the WDM sample because there are very few low mass WDM halos available to scatter up in luminosity.

The effect of this scatter on void statistics is readily seen in the void volume fraction, $f_{\text {void }}$, defined as the fraction of randomly-placed spheres that contain no mock galaxies - i.e. the fraction of the total volume occupied by empty (spherical) voids. Fig. 2 shows that the void volume fraction $\left(f_{\text {void }}\right)$ becomes significantly lower with increasing void size for CDM voids with high $L / V_{c}$ scatter.

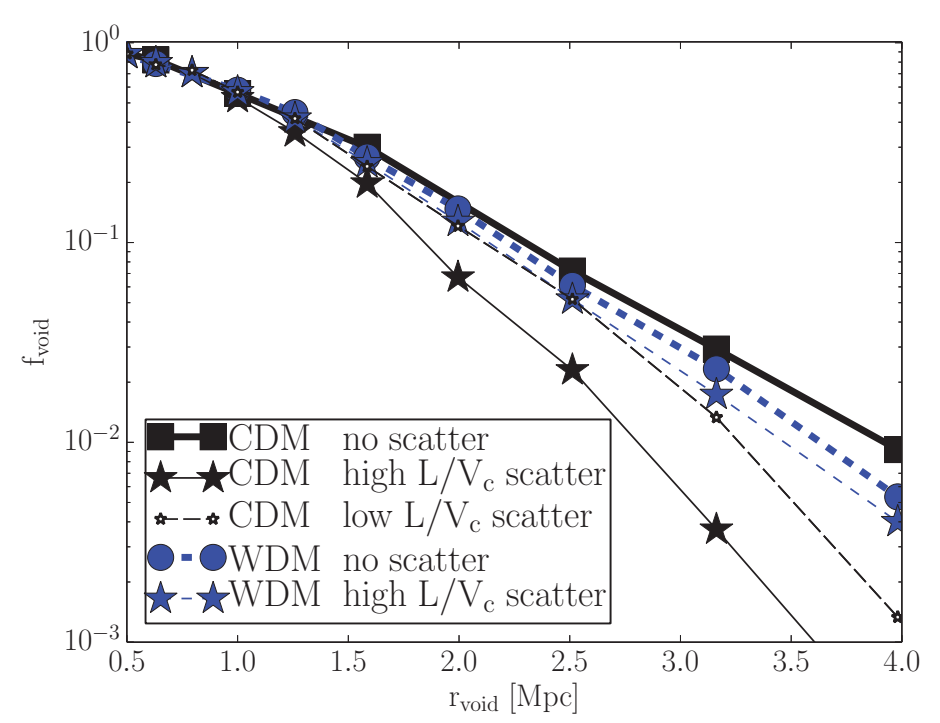

Fig. 2. The void volume fraction for the WDM and CDM mock galaxy catalogs, defined by the fraction of randomly-placed spheres that contain zero galaxies. Stars show that a large scatter between galaxy luminosity and halo $V_{c}$ (described in text), reduces the numbers of large empty CDM voids.

\section{Discussion of Luminosity Scatter in Dwarf Galaxies}

One should ask whether a large $L / V_{c}$ is expected or even plausible. For bright galaxies (lying in massive halos), one can use the clustering strength with e.g. the auto-correlation function to constrain halo masses, utilizing the fact that halos become increasingly more strongly clustered with increasing mass. However, for dwarfs, clustering strength has little constraining power on halo mass because the clustering strength of low mass halos has only weak dependence on halo mass and is very similar to that of the dark matter distribution - i.e. halos are not highly biased. Scatter for dwarfs is thus not well-constrained.

Some hints can be found from kinematic mass measurements from individual dwarfs. A large scatter between galaxy luminosity and halo mass is suggested 
by the Tully-Fisher relation for dwarfs, which exhibits increased scatter between luminosity and circular velocity for decreasing $V_{c}$ for dwarf galaxies ${ }^{10}$. Considering luminosities of even fainter galaxies, the common 300 parsec dynamical mass scale of $10^{7} h^{-1} M_{\odot}$ over $\sim 5$ orders of magnitude in luminosity for Milky Way satellites ${ }^{11}$ may reflect a very large scatter in dwarf galaxy luminosity to halo mass, though it may instead simply reflect a rapid drop in luminosity over a narrow halo mass range.

Ref. 12 cites the existence of more than expected relatively bright but isolated galaxies in the local volume as a potential CDM problem; massive halos in CDM simulations prefer to lie instead in the walls, filaments, and knots that bound void volumes. Our results may alleviate any such problem. We show that within a CDM universe that also has large halo mass to luminosity scatter, there is the potential for a population of relatively isolated and bright galaxies.

\subsection{Prospects for the future}

Sufficiently large and deep future surveys (e.g. the Large Synoptic Survey Telescope) should provide sufficient statistics to detect whether we live in a CDM universe with large scatter in $L / V_{c}$ using local volume void statistics. Large enough numbers of dwarf galaxies in small voids would rule out WDM. If it happens instead that we live in a WDM universe, such void statistics combined with an independent determination of a large $L / V_{c}$ scatter could allow CDM to be ruled out by the emptiness of small voids. Finally, we note that if the dwarf galaxy $L / V_{c}$ scatter is small, there would seem to be little potential of using the cosmic web to constrain whether we have CDM versus a WDM cosmology.

\section{Acknowledgments}

This work is largely based on the results of Reed et al. (2015) and was prepared for the Fourteenth Marcel Grossmann Meeting (2015) http: //www . icra.it/MG/mg14/. What a debt we all owe to Donald Knuth for his gift of $\mathrm{T}_{\mathrm{EX}}$ to us and to Leslie Lamport as well for its LATEX child.

\section{References}

1. Moore B., Ghigna S., Governato F., Lake G., Quinn T., Stadel J., Tozzi P., 1999, Astrophys. J. Lett. , 524, L19

2. Klypin A., Kravtsov A. V., Valenzuela O., Prada F., 1999, Astrophys. J. , 522, 82

3. Benson A. J., Bower R. G., Frenk C. S., Lacey C. G., Baugh C. M., Cole S., 2003, Astrophys. J., 599, 38

4. Tikhonov A. V., Klypin A., 2009, Mon. Not. R. Astron. Soc. , 395, 1915

5. Tikhonov A. V., Gottlöber S., Yepes G., Hoffman Y., 2009, Mon. Not. R. Astron. Soc. , 399, 1611

6. Reed D. S., Schneider A., Smith R. E.; Potter D. Stadel J., Moore B., 2015, Mon. Not. R. Astron. Soc. , 451, 4413 
7. Stadel J. G., 2001, PhD thesis, Univ. of Washington

8. Gill S. P. D., Knebe A., Gibson B. K., 2004, Mon. Not. R. Astron. Soc. , 351, 399

9. Knollmann S. R., Knebe A., 2009, Astrophys. J. Suppl. , 182, 608

10. Geha M., Blanton M. R., Masjedi M., West A. A., 2006, Astrophys. J. , 653, 240

11. Strigari L. E., Bullock J. S., Kaplinghat M., Simon J. D., Geha M., Willman B., Walker M. G., 2008, Nature, 454, 1096

12. Peebles P. J. E., Nusser A., 2010, Nature, 465, 565 